\title{
Socio-Environmental, Lifestyle, Behavioural, and Psychological Determinants of Biological Ageing: The Singapore Longitudinal Ageing Study
}

\author{
Tze Pin Ng ${ }^{a}$ b Xin Zhong ${ }^{c} \quad$ Qi Gao ${ }^{a} \quad$ Xinyi Gwee $^{a}$ Denise Qian Ling Chua ${ }^{a}$ \\ Anis Larbid, e \\ a Department of Psychological Medicine, Yong Loo Lin School of Medicine, National University of Singapore, \\ Singapore, Singapore; ${ }^{b}$ Geriatric Education and Research Institute, Ministry of Health, Singapore, Singapore; ' Social \\ and Cognitive Computing Department, Institute of High-Performance Computing, Agency for Science, Technology \\ and Research (A*STAR), Fusionopolis, Singapore, Singapore; ${ }^{d}$ Singapore Immunology Network (SlgN), Agency for \\ Science Technology and Research (A*STAR), Singapore, Singapore; e Department of Biology, Faculty of Sciences, \\ University Tunis El Manar, Tunis, Tunisia
}

\section{Keywords \\ Health span - Promoting factors - Physical function - Mental and cognitive activity · Nutrition manuscript length}

\begin{abstract}
Introduction: The identification of modifiable health spanpromoting factors is a public health priority. Objective: To explore the socio-environmental, lifestyle, behavioural, and psychological determinants of a clinical phenotypic measure of biological ageing in the Singapore Longitudinal Ageing Study (SLAS) cohort. Methods: Using cross-sectional data on 2,844 SLAS-2 adults with a chronological age (CA) $\geq 55$ years, we estimated biological age (BA) using a validated panel of clinical, biochemical, physiological, and functional indicators ( 8 in men and 10 in women) and calculated the difference between BA and CA (BA - CA in years). Potential determinants included education, housing status, loss of a spouse, living alone, lifestyle and health activity, smoking, alcohol consumption, nutritional risks, consumption of milk, soy, fruit, vegetables, coffee and tea, sleep parameters, and life satisfaction. Results: The mean CA was 67.0 (standard deviation [SD] 7.9; range 55-94) years. The estimated BA varied more widely (SD 8.9 years; range $47.5-119.9$ years), and
\end{abstract}

karger@karger.com www.karger.com/ger

Karger"

BOPEN ACCESS
(C) 2020 The Author(s)

Published by S. Karger AG, Basel

This is an Open Access article licensed under the Creative Common Attribution-NonCommercial-4.0 International License (CC BY-NC) (http://www.karger.com/Services/OpenAccessLicense), applicable to the online version of the article only. Usage and distribution for commercial purposes requires written permission.
BA - CA ranged from -11.3 to 30.0 years. In stepwise selection regression analyses, multiple significant independent determinants in a final model were larger for private housing, being single/divorced/widowed, productivity, cognitive and leisure time activity scores, $10 \mathrm{~h}$ /week of moderate-tovigorous physical activity, unintended loss of weight, life satisfaction, and daily consumption of fruits $1-2$ or $\geq 3$ servings and Chinese tea 1-2 or $\geq 3$ cups daily, together explaining $16 \%$ of BA - CA variance in men and $14 \%$ in women. Associated $B A$ - CA estimates were highest in men with high-end housing status ( -1.8 years, effect size 0.015$)$ and unintended weight loss (1.5 years, effect size 0.017). Conclusion: We identified determinants of biological ageing which can promote health span.

(C) 2020 The Author(s)

Published by S. Karger AG, Basel

\section{Introduction}

With the global population ageing, countries all over the world currently face the mounting societal challenges of dealing with the increasing burden of disease and disability resulting from a longer life expectancy. Much of the gains in life expectancy have thus far been achieved by

Tze Pin Ng

Department of Psychological Medicine, Gerontology Research Programme

National University of Singapore, NUHS Tower Block, 9th Floor

1E Kent Ridge Road, Singapore 119228 (Singapore)

pcmngtp@nus.edu.sg 
advances in medical care and public health [1] made in past decades that reduce the mortality associated with individual diseases, particularly infectious diseases, malnutrition, and cardiovascular disease, with the exception of diabetes. Currently, there is an axiomatic need for finding ways to help people stay healthy and free of disability as they age, i.e., extending a healthy life expectancy ("health span"). Given that the majority of the elderly have multiple diseases, it has been argued that the extent to which effective treatment or the prevention of individual diseases is able to improve health span is limited [2]. For this reason, it is proposed that the slowing of ageing itself is probably the most effective strategy for extending health span [3].

Research into the area of biological ageing and health span extension has accelerated at a phenomenal rate in the past decade. There is broad consensus that the molecular changes associated with ageing can be slowed or reversed in animals, resulting in an increased healthy life span. However, the clinical translation of these findings to health span extension in humans is lengthy and complex. Clinical studies investigating molecular targets (e.g., metformin, rapamycin analogues, etc.) of health span extension in humans are nascent [4]. These translational prospects are made possible by very recent important contributions to measuring biological ageing in humans.

Given that biological ageing reflects the progressive deterioration ofbiological system integrity at multiple (i.e., molecular, cellular, tissue, organ, and whole-body) levels, a number of approaches have been proposed to measure biological ageing. These measures of biological age (BA) broadly include cellular and molecular estimators such as "telomeric age" (based on leukocyte telomere length $[5,6])$, DNA methylation age ("epigenetic clocks") [7-12], physiological age (based on clinical measures and routine blood biomarkers), and functional age (cognitive age, functional age, and Frailty Index [FI] [13-16]). Studies have shown that all BA estimators correlate to varying degrees except for telomere length and are associated with mortality risk independently of chronological age (CA). Regarding different BA estimators, measures of physiological age and functional age, especially the FI, appear to be stronger and more robust predictors of mortality [17-21], although more recent algorithmic versions of DNA methylation age (DNAmGrimAge or DunedinPoAm) show similar large effects, and, jointly, DNA methylation and FI remain independent predictive of mortality [21]. Given the current state of science, it thus appears that molecular and physiological and/or functional estimators of BA reflect different ageing pheno- types and may complement each other for application in different settings.

Clinical phenotypic measures of biological ageing based on composite measurements of standard clinical chemistry and physiological and functional measures of ageing are relatively inexpensive and simple to use, making them suitable for evaluating the effects of clinical or public health interventions aimed at extending health span. Several measures of BA have been created and validated in a number of different populations [13-16] including in Asia [22-24]. Recently, we developed a population-specific measure of biological ageing among Singaporean Chinese, based on routine blood chemistry and physiological and functional biomarkers ( 8 in men and 10 in women) that robustly differentiate biologically older and younger individuals with the same CA, with respect to their future risk of physical frailty and mortality [25]. For 2 individuals with the same CA, the one who is biologically older based on his/her estimated BA is more likely to become frail or die earlier, and vice versa.

While studies of clinical interventions to extend health span are progressing apace, it has also been recently highlighted that an urgent public health priority is the identification and amelioration of modifiable lifestyle, behavioural, and environmental factors that could help to increase health span [26]. Factors such as physical activity, nutrition, and stress avoidance are most likely the sort of modifiable determinants of healthy life span, but there is a paucity of studies that have investigated and documented some but not all social and behavioural factors $[12,15$, 21, 27-29]. The aim of this study was to attempt a wideranging exploration of lifestyle, behavioural, psychologi$\mathrm{cal}$, and socio-environmental determinants of a validated clinical phenotypic measure of biological ageing that was recently reported in a previous study on the subjects and data from the Singapore Longitudinal Ageing Study (SLAS).

\section{Methods}

\section{Study Population}

The SLAS was a population-based prospective cohort study on the ageing and health of middle-aged and older adults (i.e., $\geq 55$ years) in Singapore. Details of the SLAS are available in a previous publication [30]. The extensive range of baseline data collected from questionnaire interviews, clinical and performance-based testing and examinations, and blood sampling, included psychosocial, lifestyle, behavioural, medical, biological, physiological, dietary and nutritional, physical and neurocognitive functioning, and health status variables. This study analysed data on 2,844 Chinese adults aged 55-94 years in the second cohort (SLAS-2), who 


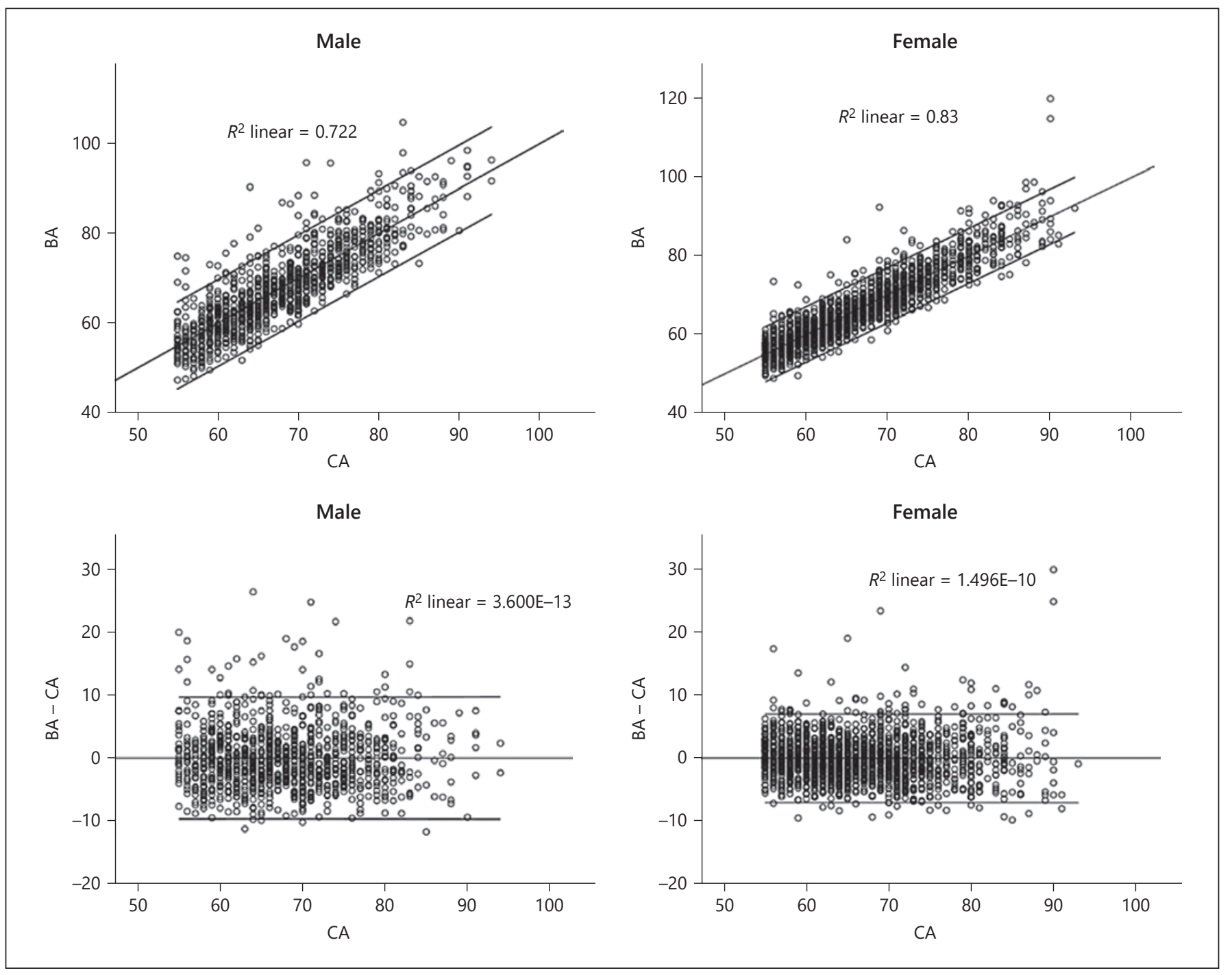

Fig. 1. Correlations between Klemera-Doubal (KM) estimates of biological age (BA) and chronological age (CA).

were recruited from March 2008 to November 2013. As reported in our previous publication, the clinical phenotypic measure, BA, was developed and validated through follow-up re-interviews and assessments of incident physical frailty at intervals of 4-5 years from January 2013 to August 2018, and of mortality up to December 2016. We analysed the baseline data on lifestyle, behavioural, psychological, and socio-environmental determinants and biological ageing in this cross-sectional study.

\section{Clinical Phenotypic BA Measure}

We used a sex-specific measure of BA derived from and validated in the SLAS cohort, as described previously [25]. The biomarkers were based on routine clinical measurements of blood, physiological, and functional biomarkers that are commonly available in clinical practice. They were selected from a total of 68 (32 blood or serum; 36 clinical and functional) different biomarkers by initially identifying those that were significantly associated with CA
( $r \geq 0.2, p<0.01)$, and then removing those which were highly associated with other biomarkers but with a relatively small contribution to the estimation of age. Stepwise selection, multiple linear regression modelling resulted in the final selection of 8 markers for males, covering physiological functioning in 6 domains of vascular function (sitting diastolic blood pressure); lung function $\left(\mathrm{FEV}_{1}\right)$, physical function (handgrip strength, knee extension strength, timed up and go, and gait speed), haematological status (haemoglobin level), kidney function (estimated glomerular filtration rate [eGFR]), and cognition (Mini-Mental State Examination [MMSE] score). For females, 10 markers were selected that covered physiological functioning in 6 domains: body composition (body massheight and thigh circumference), lung function $\left(\mathrm{FEV}_{1}\right)$, physical function (handgrip strength, knee extension strength, timed up and go, gait speed, and chair rise), haematological status (lymphocyte count), kidney function (eGFR), and cognition (MMSE score). The computation for estimating BA included the Klemera-Doubal 
Table 1. Chronological and biological age of 2,844 SLAS-2 Chinese cohort participants; demographic, socio-environmental, lifestyle, behavioural, psychological, and nutritional determinants

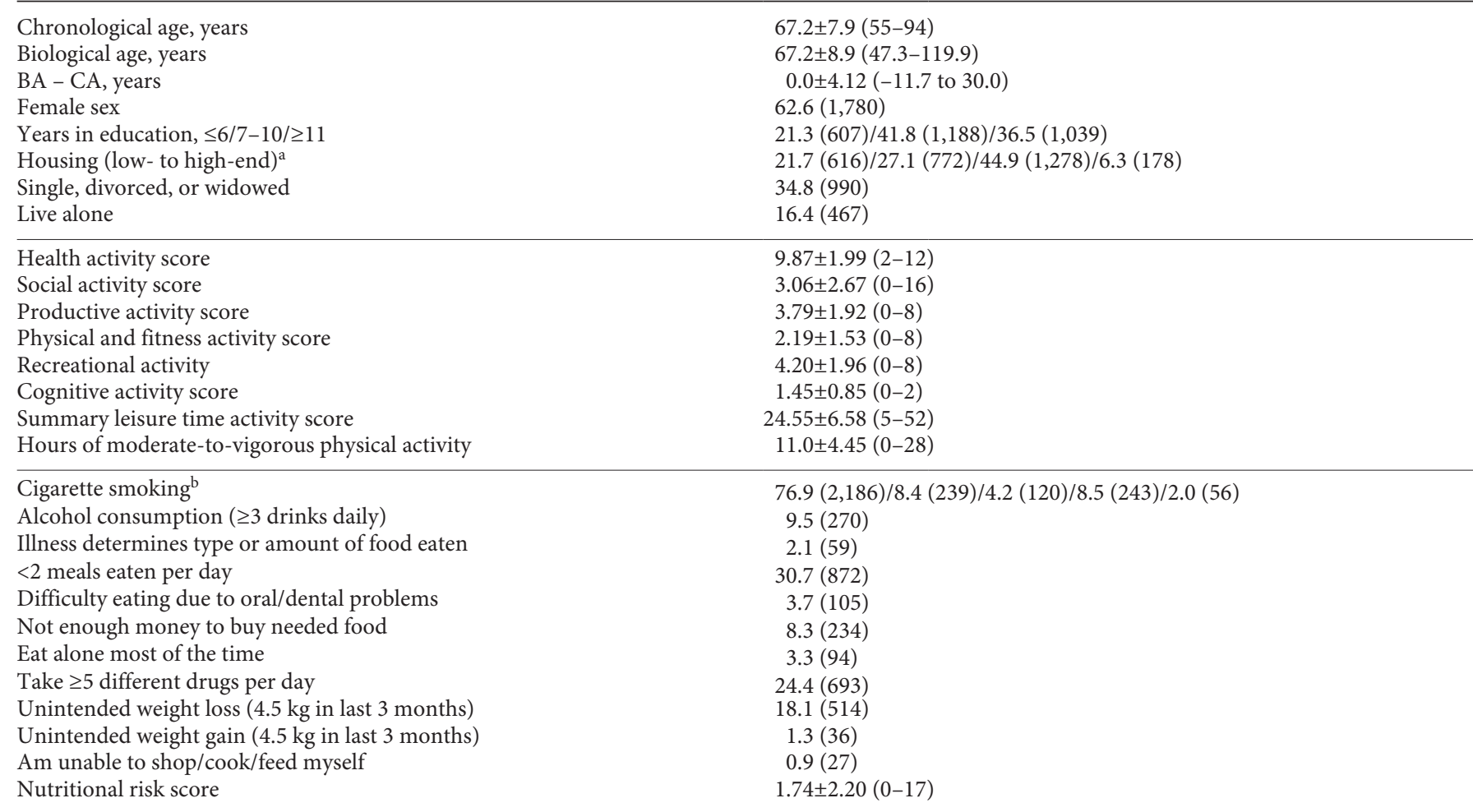

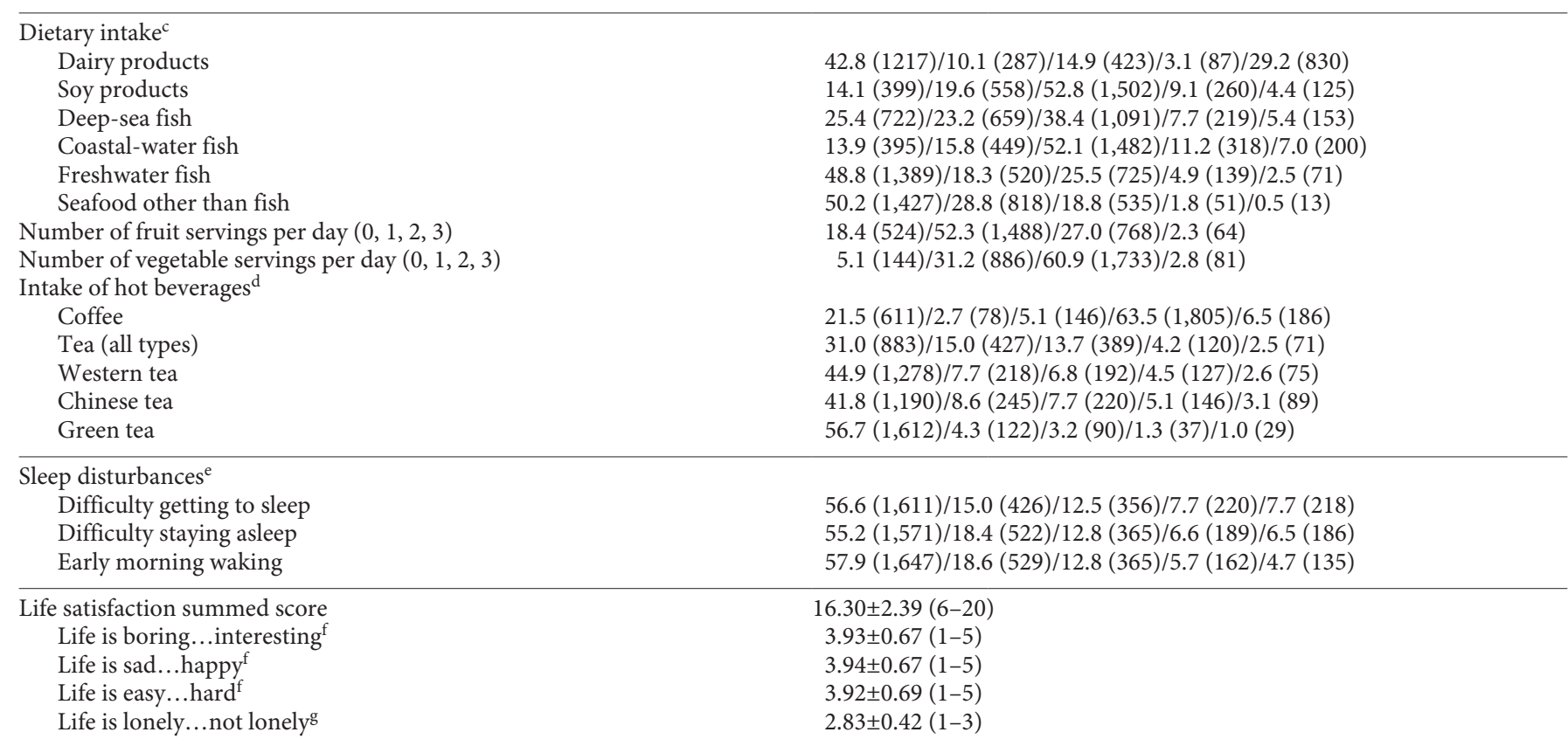

Values are expressed as $\%(n)$ or mean \pm standard deviation (min-max score).

${ }^{\text {a }} 1$ - or 2-room public housing/3-room public housing/4- or 5-room public housing/private condominiums/landed houses.

${ }^{\mathrm{b}}$ Never-smoker/ex-smoker $<20$ per day/ex-smoker $\geq 20$ per day/current $<20$ per day/current $\geq 20$ per day.

${ }^{c}$ Never or rarely/more than once per week/1-3 times per week/4-6 times/week/daily.

d Never or rarely $/<1$ cup per week $/ \geq 1$ cup per week to $<1$ cup daily $/ 1-2$ cups daily $/ \geq 3$ cups daily.

${ }^{\mathrm{e}} \mathrm{Never} /$ rarely $(<1 \times$ per month)/sometimes $(\geq 1 \times$ per month $<1 \times$ per week)/frequently $(\geq 1 \times$ per week, not daily)/daily.

${ }^{\mathrm{f}}$ A scale of $1-5$, where $1=$ very, $2=$ fairly, $3=$ neither, $4=$ fairly, and $5=$ very.

g A scale of $1-3$, where $1=$ very, $2=$ fairly, and $3=$ not at all. 
(KD) algorithm, which was found to be more optimal than Principal Component Analysis, Multiple Linear Regression, and Machine Learning methods for predicting mortality and frailty. The KD estimator of BA was found to be significantly better than CA in predicting mortality and frailty (C-statistics ranging from 0.70 to 0.79 in the middle-aged group), thus supporting its relevance and usefulness for measuring health span in middle-aged individuals. The estimated BA for each individual varied by as much as 10-30 years above and below the mean (2SD) for each value of CA from 55 to 94 years. In this study, we used the residual index, BA -CA, to denote whether a person was biologically younger (a negative value in years) or older (a positive value in years) than their CA (Fig. 1).

\section{Determinants of $B A$}

Socio-Environmental Variables. These included measures of socio-economic status: years in education ( $\leq 6,7-10, \geq 11$ years), housing status (lower- to higher-end, i.e., public housing ( 1 or 2 , 3 , and 4 or 5 rooms, a private condominium or apartments, and property with land), and measures of social isolation (single/divorced/widowed and living alone).

Health and Lifestyle Activities. Health activity was measured using a summed Likert score of frequency of engagement, i.e., $1=$ never (less than once a month); 2 = sometimes (once a month or more but less than once a week); 3 = often (once a week or more), in health-related activities, namely, watch what you eat, exercise regularly (i.e., 2-3 times a week), good sleep habits, make time for leisure and relaxation, and habits regarding smoking and alcohol consumption.

Lifestyle and Leisure Time Activity. These were measured for 8 categories of social activities (attending religious worship, sport events, excursions, card games, seniors' clubs, and group singing or dancing), 5 categories of productive activities (hobbies, cookery, shopping, volunteerism, and paid or unpaid employment), 3 categories of physical and fitness activities (brisk walking, exercise routines, and sports), recreational activities (television, radio, music, computer games), and 1 category of cognitively stimulating activities (reading, drawing, painting, photography, attending courses, puzzles, mind games, etc.). Summed Likert scores of the frequency of participation (as above) for each domain of social, productive, physical and fitness, recreational, and cognitive activities and a total summary score of health and lifestyle activities was computed. Additionally, the estimated total number of hours of participation in moderate- and vigorous-level physical activities was reported by the respondents.

Sleep Health. Respondents reported the frequency ( 1 = never; $2=$ rarely, i.e., $<1 \times /$ month; $3=$ sometimes, i.e., $\geq 1 \times /$ month but $<1 \times /$ week, 4 = frequently, i.e., $\geq 1 \times /$ week but not daily, $5=$ always, i.e., daily, of disturbed sleep (difficulty getting to sleep or staying asleep and early-morning waking).

Life Satisfaction. This was measured using Likert response scores to 3 questions on whether the respondent finds his/her life to be boring or interesting, sad or happy, hard or easy on a scale of 1-5 ( 1 = very boring, 2 = fairly boring, $3=$ neither, $4=$ fairly interesting, $5=$ very interesting) and 1 question on whether the respondent felt lonely ( 1 = very lonely, 2 = fairly lonely, $3=$ not at all lonely). A summed score of total life satisfaction was derived.

Smoking. Participants reported their smoking history and were categorized as $1=$ never-smokers, $2=$ ex-smoker of $<20$ cigarettes/ day, 3 = ex-smoker of $\geq 20$ cigarettes/day, 4 = current smoker of $<20$ cigarettes/day, $5=$ current smoker of $\geq 20$ cigarettes/day.
Alcohol. Self-reported history of alcohol consumption was used to categorize participants by their level of drinking, i.e., $\geq 3$ drinks a day $(=1)$ or less $(=0)$.

Nutritional Risk. We used the DETERMINE Your Nutritional Health check-list, developed for the Nutrition Screening Initiative by the American Dietetic Association, the American Academy of Family Physicians, and the National Council on Ageing in the USA, to assess the risk of malnutrition in older adults. The checklist includes 10 Yes/No question-items describing personal and behavioural factors related to inadequate or poor-quality food and nutrient intake among older people (Table 1). Weighted scores for individual responses are summed to give a total score ranging from 0 to 21 ; a score of $\geq 6$ indicates a high nutritional risk, 3-5 a moderate nutritional risk, and $0-2$ a good nutritional status.

Additionally, participants gave their responses on the frequency of specific intake of milk and dairy products, soy products, fish (deep-water, coastal, fresh-water, and other seafood): $1=$ never or rarely, $2=<$ once/week, $3=1-3$ times/week, $4=4-6$ times/week, $5=$ daily. Consumption of fruit and vegetables was quantitated by the number of servings $(0,1$, or $2+)$ consumed per day. The frequency of coffee and tea consumption (Chinese black and oolong tea, green tea, and Western black tea drunk with milk) was quantitated as $1=$ never or rarely, $2=<1 \mathrm{cup} /$ week, $3=\geq 1 \mathrm{cup} /$ week to $<1$ cup/day, $4=1-2$ cups/day or $5=\geq$ cups/day, and the equivalent frequency and amount of tea of all types was computed.

Disease and Disability. The presence and number of chronic diseases in individual participants were ascertained by self-reports of a physician diagnosis and treatment for 18 common diagnoses and others in the questionnaire, corroborated by the recording of names of drugs on medication packages produced by the participants as well as measurements of their blood pressure, fasting blood glucose, lipids, and eGFR. For example, hypertension included known cases of anti-hypertensive treatment as well as unknown or untreated cases with raised systolic or diastolic blood pressure based on standard clinical criteria. Raised fasting blood glucose was used define prediabetes or undiagnosed diabetes, and eGFR $<60 \mathrm{~mL} / \mathrm{min} / 1.73 \mathrm{~m}^{2}$ to define chronic kidney disease, according to standard clinical criteria. The number of comorbidities was estimated from the total count of medical disorders in the past year. Disability was measured by dependency when performing the basic and instrumental activities of daily living used in the Barthel and the Lawton indexes.

\section{Statistical Analysis}

We used regression analyses to estimate the associations of individual determinant variables with residual BA - CA as the normally distributed continuous dependent variable. The total of 49 potential determinants included untransformed continuous variables that met assumption of normal distribution and were tested for linear relationships with $\mathrm{BA}-\mathrm{CA}$, and ordinal variables that were analysed categorically with ANOVA testing for non-linear relationships (online supplementary Tables S1 and S2; for all online suppl. material, see www.karger.com/doi/10.1159/000511211). Mean BA - CA estimates from multiple group comparisons were made with the Bonferroni correction (95\% confidence interval [CI]). A forward stepwise selection approach was used to identify significant determinants in a final model of multiple independent determinants that are significant in the presence of each other using probability at entry of $p<0.05$ and at removal of $p<0.10$. The results were presented separately for men and women in stratified analyses by sex. 
Table 2. Regression estimates of BA - CA associated with significant determinants in men and women

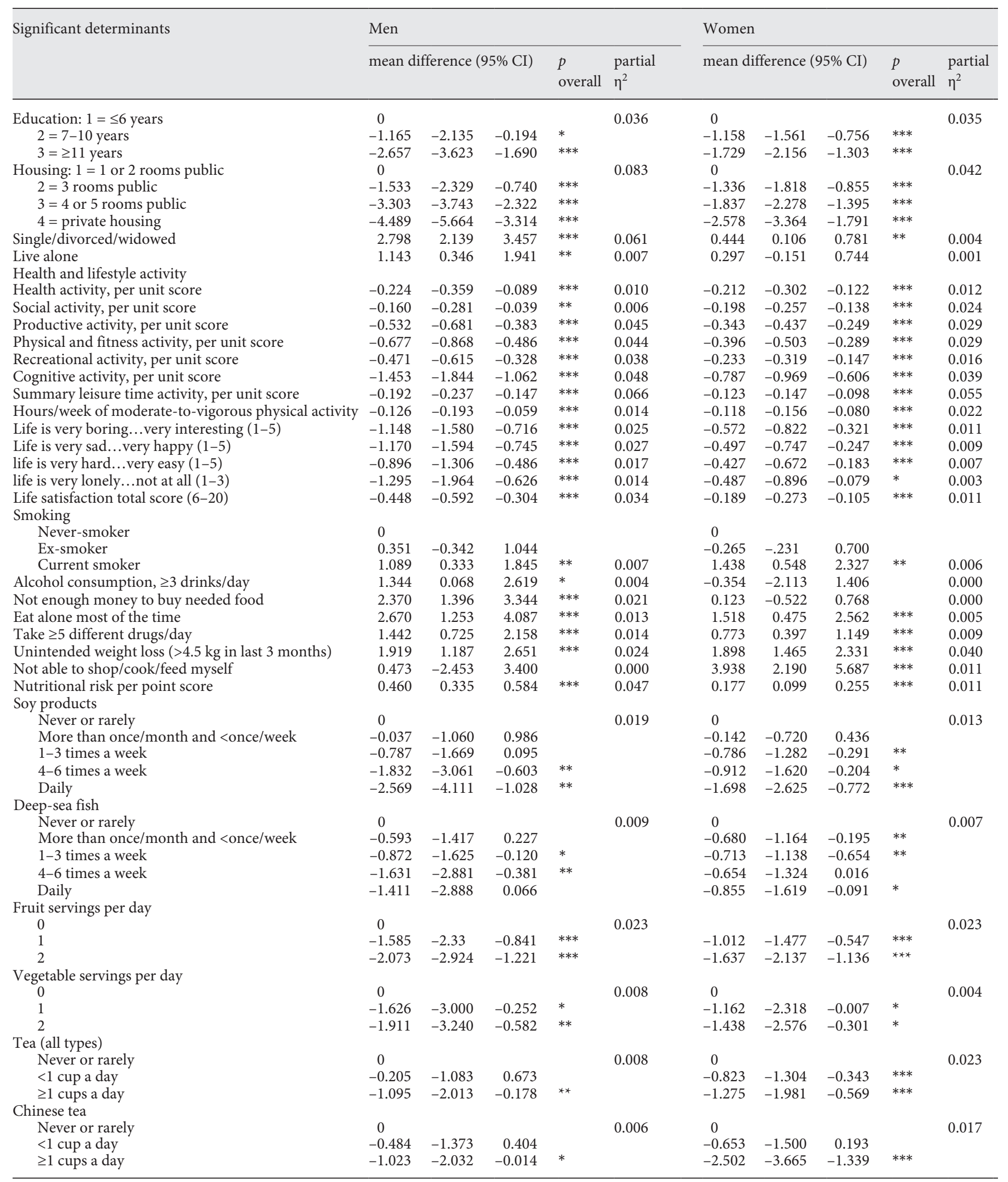


Table 3. Significant lifestyle variables in final stepwise selection model of BA - CA years in men and women

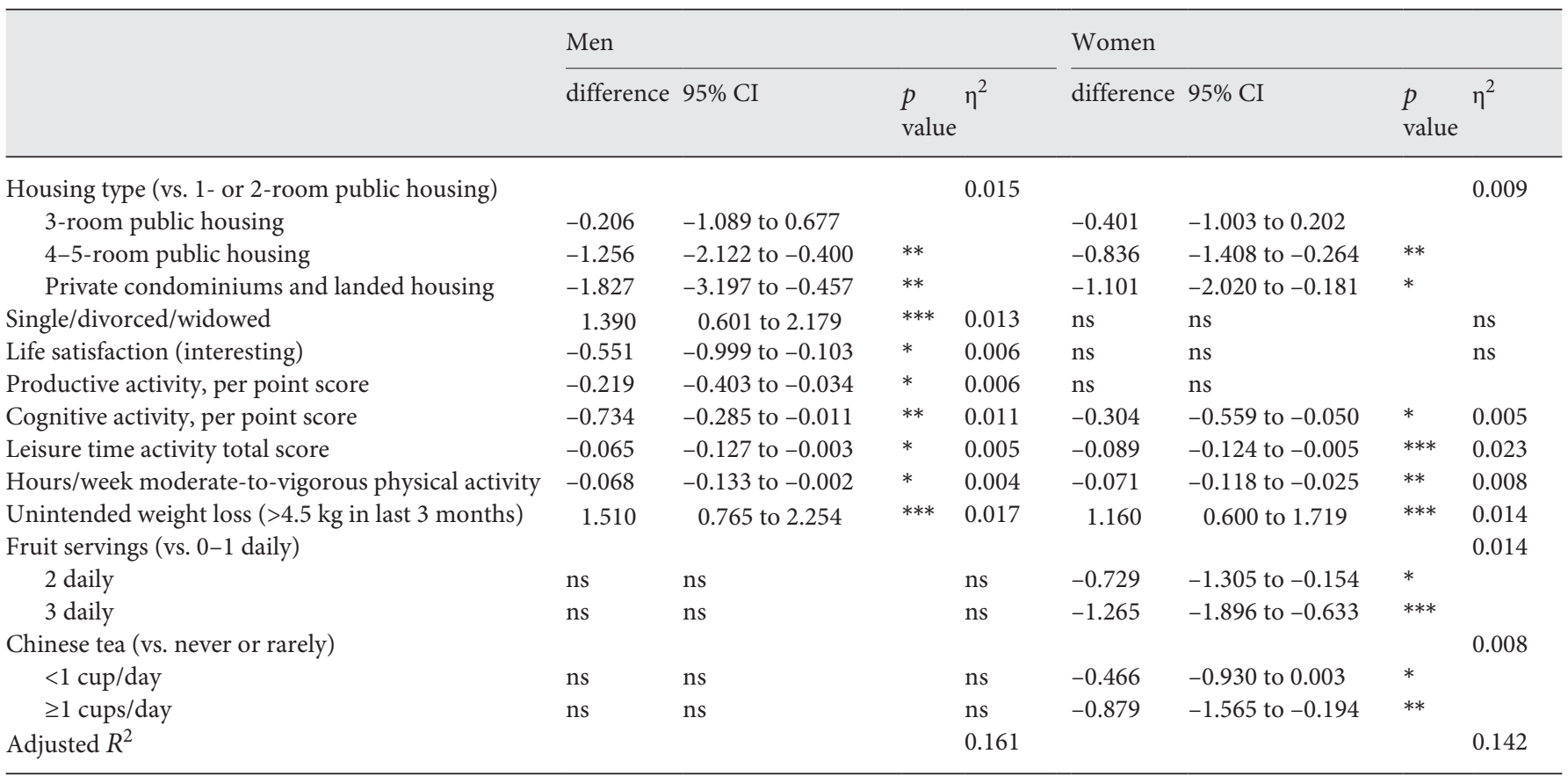

\section{Results}

The mean CA of the cohort was 67.0 (SD 7.9; range $55-94)$ years. The estimated BA varied more widely (SD 8.9 years), ranging from 47.5 to 119.9 years, and was higher for men than women (mean 67.8 [SD 9.3] and 66.7 [SD 8.7], respectively). As indicated by the BA - CA residuals, the $\mathrm{BA}$ for individuals varied by as much as 11 years below and 30 years above the CA (Table 1; Fig. 1). There was no difference in BA - CA years between men and women.

Estimates of the association of the BA - CA residuals with all 49 possible determinants as shown by the mean differences and $95 \%$ CI of BA - CA in years between groups or per point scores are listed in online supplementary Tables S1 and S2. Determinants which showed statistically significant $(p<0.05)$ associations with BA - CA years in men and women are shown in Table 2 . For example, more years in education $(7-10$ years and $\geq 11$ years relative to $\leq 6$ years) were associated significantly ( $p<$ 0.001 ) with BA - CA estimates of -1.16 and -2.66 years, respectively, in men, and -1.16 and -1.73 years, respectively, in women, suggesting that more highly educated individuals are more likely to be biologically younger by 1-2 years. Other variables that were significantly associated with a younger BA were higher levels of education and better housing, health and lifestyle activities, life satisfaction, and a diet including the consumption of soy products, deep-sea fish, fruit and vegetables, and tea. Variables that were significantly associated with older BA were being single, divorced, or widowed, living alone, smoking, alcohol consumption, having a higher nutritional risk, and a poor nutritional status.

In the stepwise selection regression analyses, independent determinants in a final model which remained significant in the presence of each other were: better-quality housing, being single, divorced, or widowed, and life satisfaction (in men); and being productive, engaging in cognitive activity, leisure activities, the number of hours per week of moderate-to-vigorous physical activity, unintended loss of weight, daily fruit consumption, and drinking Chinese tea (in women) (Table 3).

The estimates of BA - CA associated with these significant variables of interest were further adjusted for the presence of disability and the number of co-occurring chronic diseases as forced-entry covariates in expanded models. The adjusted BA - CA estimates associated with lifestyle, behavioural, and socio-environmental variables of interest were attenuated when controlled for the presence of disease and disability, but basically remained significantly associated with BA - CA (Table 4). 
Table 4. Significant lifestyle variables in final stepwise selection model of BA - CA years in men and women, adjusted additionally for number of comorbidities and disability

\begin{tabular}{|c|c|c|c|c|c|c|c|c|}
\hline & \multicolumn{4}{|l|}{ Men } & \multicolumn{4}{|l|}{ Women } \\
\hline Housing type (vs. 1- or 2-room public housing) & & & & 0.007 & & & & 0.011 \\
\hline 3-room public housing & -0.554 & -1.395 to 0.287 & & & -0.441 & -1.036 to 0.154 & & \\
\hline 4-5-room public housing & -1.596 & -2.412 to -0.780 & $* * *$ & & -0.883 & -1.446 to -0.320 & $* *$ & \\
\hline Life satisfaction (interesting) & -0.455 & -0.880 to -0.029 & $*$ & 0.004 & ns & ns & & ns \\
\hline Productive activity, per point score & -0.249 & -0.401 to -0.097 & $*$ & 0.010 & ns & ns & & \\
\hline Cognitive activity, per point score & -0.777 & -1.181 to -0.373 & $* * *$ & 0.014 & ns & ns & & 0.005 \\
\hline Leisure time activity total score & ns & ns & & ns & -0.090 & -0.122 to -0.058 & $* * *$ & 0.027 \\
\hline Hours/week moderate-to-vigorous physical activity & ns & ns & & ns & -0.052 & -0.099 to -0.005 & $*$ & 0.004 \\
\hline Chinese tea (vs. never or rarely) & & & & & & & & 0.007 \\
\hline$<1$ cup/day & ns & ns & & ns & -0.393 & -0.855 to 0.069 & & \\
\hline$\geq 1$ cups/day & ns & ns & & ns & -0.852 & -1.532 to -0.172 & $* *$ & \\
\hline Adjusted $R^{2}$ & & & & 0.148 & & & & 0.159 \\
\hline
\end{tabular}

The effect sizes estimated by the partial $\eta^{2}$ were small for individual determinants and were highest for housing status $(1.5 \%)$ and unintended weight loss (1.7\%). The final model of all significant determinants of BA - CA had an $R^{2}$ value of $16 \%$ in men and $14 \%$ in women, suggesting remaining unexplained variances in BA - CA due to unmeasured factors.

We estimated the combined effects of significant determinants of $\mathrm{BA}$ in the individual by creating a Health Span Factors Score (HSFS), i.e., a summed score of binary and ordinal integer scores $(0,1$, and 2$)$ for each of the significant predictors, with higher scores denoting a positive valence value of health span, except for weight loss which was assigned a negative value of -1 . The mean HSFS of the participants was 8.0 (SD 2.9; range 1-15). Figure 2 shows the mean and $95 \%$ CI of BA - CA in years associated with HSFS, showing a linear declining trend from 3.5 years (2.5-4.6) for an HSFS of 1 to -2.5 years $(-3.9$ to -1.1$)$ for an HSFS of 15.

\section{Discussion}

To achieve the goal of enhancing the well-being of individuals as they age, the factors that determine the trajectories of ageing need to be better understood. Our study reveals a larger number of lifestyle, behavioural, and socio-environmental factors are linked to biological ageing than previously thought, and provides vital insights into potentially modifiable determinants of healthy life span for targeted interventions. In retrospect, the results are probably not surprising, as the same health risk and protective factors are also known determinants of chronic diseases and disability.

The clinical phenotypic measure of biological ageing used in this study represents organ-system level physiological and functional manifestations of multiple cellular and molecular processes of ageing. The variations in biological ageing, as such, that are explained by health risk variables are posited to be mediated by cellular processes of biological ageing like epigenetic modifications, telomere erosion, mitochondrial energy balance dysregulation, and inflammation. There are cellular-level measures of biological ageing such as epigenetic, telomere, mitochondrial DNA, inflammation, and "omics" "clocks" that we did not use in this study. Currently, there is still much to understand about the inter-relationships of clinical and molecular measures of BA, whether they measure the same or different processes of biological ageing, and their causal roles in the ageing process and diseases. Recent studies suggest that the correlations between extant clini- 


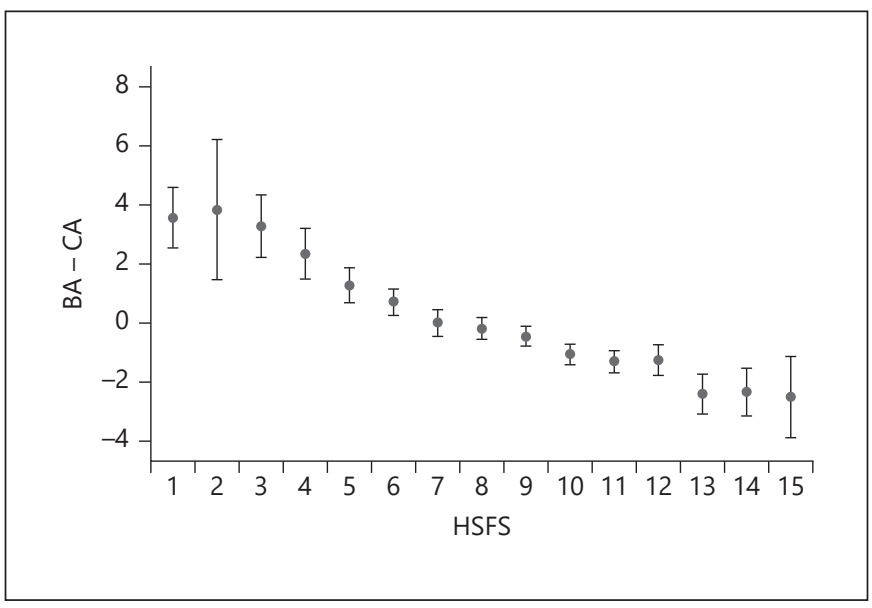

Fig. 2. Mean and $95 \% \mathrm{CI}$ estimates of BA - CA in years for integer values of Health Span Factors Score (HSFS). Linear regression: BA - CA $3.819-0.476 \times$ HSF. HSFS is the summed score of education $(0,1,2)$, housing status $(0,1,2,3)$, productive activity $(0,1,2)$, physical and fitness activity $(0,1,2)$, cognitive activity $(0,1), \geq 10$ $\mathrm{h}$ /week of moderate to vigorous activities $(0,1)$, daily fruit servings $(0,1,2)$, tea consumption $(0,1,2)$, life very interesting $(0,1)$, and loss of weight $(0,-1)$. Cutoff values are as shown in the Tables. Productive activity cutoffs were $0-2,3-4,5-10$; physical and fitness activity cutoffs were $0-1,2$, and $3-8$.

cal biomarkers and cellular or molecular biomarker measures of ageing (in particular, the epigenetic clock and telomere length) are, in general, weak, suggesting that they quantify different processes of biological ageing [1721].

Our study adds to a number of recent studies, exploring the socio-environmental and health-related determinants of health span, that have used molecular or composite physiological clinical biomarkers of biological ageing. These studies also reported similar relationships with summary measures of life-course risk factors including low educational attainment, material and social resource deficits, and mental health problems [12, 15, 19, 21, 2729]. Notably, an early personal and childhood history of familial longevity, social class, adverse experiences, and health, intelligence, and self-control were shown to predict more rapid physiological ageing (using the Pace of Ageing Index) [27]. A low level of education and smoking, relatively more than other lifestyle-related risk factors such as alcohol intake or obesity, were also shown to be associated with accelerated epigenetic ageing [28]. In another recent study, health-related lifestyle behaviours (obesity, smoking, alcohol consumption, and physical activity) exhibited the greatest contribution to the Pheno-
Age Index of biological ageing, closely followed by adversity in adulthood [29].

In line with previous studies, our study provides further evidence in support of the predominant influence of socio-environmental circumstances and lifestyle behavioural factors on biological ageing. Socio-economic factors reflecting access to material and social resources appeared as the strongest determinants of health span, and physical and cognitive activities stand out as amenable behavioural factors. In addition, our study revealed other lifestyle behavioural factors such as the consumption of fruit and tea, which have not been reported in previous studies. Their estimated associations are robust to the statistical control of mutual confounding by multiple covariates in the models and also consistent with established knowledge about their influence on health and disease. For example, tea which is rich in polyphenols such as epigallocatechin-3-gallate (EGCG) with powerful antioxidant and anti-inflammatory actions, is regarded as an "anti-senescence food" for healthy ageing [31, 32].

The effect sizes associated with individual determinants were small but can, collectively, contribute substantially to extending health span in real-world settings. The HSFS of the individual, reflecting the number and intensity of health span-promoting factors, suggest that, for those in the highest-percentile group, BA may be younger than CA by $\geq 2$ years at the population level (and some individuals may even be $\geq 10$ years younger). Interestingly, this is consistent with the estimate that delaying ageing could increase population life expectancy by an additional 2.2 years, most of which would be spent in good health [2].

The effect size estimates of these determinants may be underestimated because of technical limitations in their measurements, which were derived from self-reports. Another limitation is that our BA estimator derived from the SLAS-2 cohort have yet to be further validated externally in an independent replication cohort. Because of the cross-sectional design of the study, the presence of prevalence or survival bias and the inability to fully capture exposure over a lifetime should be considered. Disentangling the relations between disease, disability, and biological ageing is difficult. Although health risk determinants were mostly found to remain significantly associated with the BA - CA measure of biological ageing after controlling for the number of comorbid chronic diseases and disability, cross-sectional analysis cannot establish causal relationships. Few longitudinal studies have investigated whether accelerated biological ageing 
resulted from pre-existing diseases, or if the biological ageing was a risk factor prior to midlife that preceded disease onset. One study on young adults, the Dunedin birth cohort study, demonstrated that individuals showing rapid biological ageing before midlife were more susceptible to the effects of disease and physical and cognitive decline [14].

Other potential determinants of health span that were not examined in this study should be investigated in future studies. Another limitation is that the study population consisted of middle-aged and older adults, thus excluding younger adults who should also be targeted for preventive interventions to promote healthy ageing. Further studies should also aim at combining person-level measures with cellular-level measures of biological ageing to better understand the underlying biological mechanisms for extending health span. Finally, interventional studies using these surrogate measures of biological ageing are needed to demonstrate the efficacy of lifestyle behavioural interventions in extending human health span.

\section{Acknowledgment}

We thank the following voluntary welfare organizations for their support: Geylang East Home for the Aged, Presbyterian Community Services, St Luke's Eldercare Services, Thye Hua Kwan Moral Society (Moral Neighbourhood Links), Yuhua Neighbourhood Link, Henderson Senior Citizens' Home, NTUC Eldercare Co-op Ltd, Thong Kheng Seniors Activity Centre (Queenstown Centre) and Redhill Moral Seniors Activity Centre.

\section{Statement of Ethics}

The research complied with the guidelines for human studies and it was conducted ethically in accordance with the Human Biomedical Research Acts (HBRA) of Singapore. All study participants gave their written informed consent and the study was approved by the National University of Singapore Institutional Review Board.

\section{Conflict of Interest Statement}

All authors declare there are no real or potential conflicts of interest. The sponsors had no role in the conduct of the study or preparation of this manuscript.

\section{Funding Sources}

The study was supported by research grants from the Agency for Science Technology and Research (A*STAR) Biomedical Research Council (BMRC/08/1/21/19/567) and the National Medical Research Council (NMRC/1108/2007; NMRC/CIRG/ 1409/2014).

\section{Author Contributions}

T.P.N. had full access to all of the data in the study and has primary responsibility for final content. T.P.N. reviewed the literature, designed the study, drafted and revised the manuscript. X.Z. and T.P.N. analysed the data. X.Z., Q.G., X.G., D.Q.L.C., and A.L. contributed to the study design and data collection. All authors reviewed the results and drafts, and approved the final manuscript.

\section{References}

1 Christensen K, Doblhammer G, Rau R, Vaupel JW. Ageing populations: the challenges ahead. Lancet. 2009 Oct;374(9696):1196-208.

2 Goldman DP, Cutler D, Rowe JW, Michaud PC, Sullivan J, Peneva D, et al. Substantial health and economic returns from delayed aging may warrant a new focus for medical research. Health Aff (Millwood). 2013 Oct; 32(10):1698-705.

3 Burch JB, Augustine AD, Frieden LA, Hadley E, Howcroft TK, Johnson R, et al. Advances in geroscience: impact on healthspan and chronic disease. J Gerontol Series A. 2014 Jun; 69 Suppl 1:S1-3.

4 Kaeberlein M, Rabinovitch PS, Martin GM. Healthy aging: the ultimate preventative medicine. Science. 2015 Dec;350(6265):1191-3.

5 Sebastiani P, Thyagarajan B, Sun F, Schupf N, Newman AB, Montano M, et al. Biomarker signatures of aging. Aging Cell. 2017 Apr; 16(2):329-38.
6 Benetos A, Okuda K, Lajemi M, Kimura M, Thomas F, Skurnick J, et al. Telomere length as an indicator of biological aging: the gender effect and relation with pulse pressure and pulse wave velocity. Hypertension. $2001 \mathrm{Feb}$; 37(2 Pt 2):381-5.

7 Horvath S. DNA methylation age of human tissues and cell types. Genome Biol. 2013; 14(10):R115.

8 Chen BH, Marioni RE, Colicino E, Peters MJ, Ward-Caviness CK, Tsai PC, et al. DNA methylation-based measures of biological age: meta-analysis predicting time to death. Aging (Albany NY). 2016 Sep;8(9):1844-65.

9 Horvath S, Gurven M, Levine ME, Trumble BC, Kaplan H, Allayee H, et al. An epigenetic clock analysis of race/ethnicity, sex, and coronary heart disease. Genome Biol. 2016 Aug; 17(1):171.
10 Marioni RE, Shah S, McRae AF, Chen BH, Colicino E, Harris SE, et al. DNA methylation age of blood predicts all-cause mortality in later life. Genome Biol. 2015 Jan;16(1):25.

11 Marioni RE, Shah S, McRae AF, Ritchie SJ, Muniz-Terrera G, Harris SE, et al. The epigenetic clock is correlated with physical and cognitive fitness in the Lothian Birth Cohort 1936. Int J Epidemiol. 2015 Aug;44(4):138896.

12 Levine ME, Lu AT, Quach A, Chen BH, Assimes TL, Bandinelli S, et al. An epigenetic biomarker of aging for lifespan and healthspan. Aging (Albany NY). 2018 Apr;10(4): 573-91.

13 Levine ME. Modeling the rate of senescence: can estimated biological age predict mortality more accurately than chronological age? J Gerontol A Biol Sci Med Sci. 2013 Jun;68(6): $667-74$ 
14 Belsky DW, Caspi A, Houts R, Cohen HJ, Corcoran DL, Danese A, et al. Quantification of biological aging in young adults. Proc Natl Acad Sci USA. 2015 Jul;112(30):E4104-10.

15 Liu Z, Kuo PL, Horvath S, Crimmins E, Ferrucci $L$, Levine $M$. A new aging measure captures morbidity and mortality risk across diverse subpopulations from NHANES IV: A cohort study. PLoS Med. 2018 Dec 15(12):e1002718.

16 Mitnitski AB, Mogilner AJ, Rockwood K. Accumulation of deficits as a proxy measure of aging. ScientificWorldJournal. 2001 Aug;1: 323-36.

17 Belsky DW, Moffitt TE, Cohen AA, Corcoran DL, Levine ME, Prinz JA, et al. Eleven Telomere, Epigenetic Clock, and BiomarkerComposite Quantifications of Biological Aging: Do They Measure the Same Thing? Am J Epidemiol. 2018 Jun;187(6):1220-30.

18 Murabito JM, Zhao Q, Larson MG, Rong J, Lin H, Benjamin EJ, et al. Measures of Biologic Age in a Community Sample Predict Mortality and Age-Related Disease: The Framingham Offspring Study. J Gerontol A Biol Sci Med Sci. 2018 May;73(6):757-62.

19 Hastings WJ, Shalev I, Belsky DW. Comparability of biological aging measures in the $\mathrm{Na}$ tional Health and Nutrition Examination Study, 1999-2002. Psychoneuroendocrinology. 2019 Aug; 106:171-8.

20 Li X, Ploner A, Wang Y, Magnusson PK, Reynolds C, Finkel D, et al. Longitudinal trajectories, correlations and mortality associations of nine biological ages across 20-years follow-up. eLife. 2020 Feb;9:e51507.
21 Belsky DW, Caspi A, Arseneault L, Baccarelli A, Corcoran DL, Gao X, et al. Quantification of the pace of biological aging in humans through a blood test, the DunedinPoAm DNA methylation algorithm. eLife. 2020 May;9:e54870.

22 Bae CY, Kang YG, Piao MH, Cho B, Cho KH, Park YK, et al. Models for estimating the biological age of five organs using clinical biomarkers that are commonly measured in clinical practice settings. Maturitas. 2013 Jul; 75(3):253-60.

23 Bai X, Han L, Liu Q, Shan H, Lin H, Sun X, et al. Evaluation of biological aging process - a population-based study of healthy people in China. Gerontology. 2010;56(2):129-40.

24 Yoo J, Kim Y, Cho ER, Jee SH. Biological age as a useful index to predict seventeen-year survival and mortality in Koreans. BMC Geriatr. 2017 Jan;17(1):7.

25 Zhong X, Lu Y, Gao Q, Nyunt MSZ, Fulop T, Monterola CP, et al. Estimating Biological Age in the Singapore Longitudinal Aging Study. J Gerontol A Biol Sci Med Sci. 2020 Sep;75(10):1913-20.

26 Newman AB, Kritchevsky SB, Guralnik JM, Cummings SR, Salive M, Kuchel GA, et al. Accelerating the Search for Interventions Aimed at Expanding the Health Span in Humans: The Role of Epidemiology. J Gerontol A Biol Sci Med Sci. 2020 Jan;75(1):77-86.
27 Belsky DW, Caspi A, Cohen HJ, Kraus WE, Ramrakha S, Poulton R, et al. Impact of early personal-history characteristics on the Pace of Aging: implications for clinical trials of therapies to slow aging and extend healthspan. Aging Cell. 2017 Aug;16(4):644-51.

28 Fiorito G, McCrory C, Robinson O, Carmeli C, Rosales CO, Zhang Y, et al.; BIOS Consortium; Lifepath consortium. Socioeconomic position, lifestyle habits and biomarkers of epigenetic aging: a multi-cohort analysis. $\mathrm{Ag}$ ing (Albany NY). 2019 Apr;11(7):2045-70.

29 Liu Z, Chen X, Gill TM, Ma C, Crimmins EM Levine ME. Associations of genetics, behaviors, and life course circumstances with a novel aging and healthspan measure: Evidence from the Health and Retirement Study. PLoS Med. 2019 Jun;16(6):e1002827.

30 Niti M, Yap KB, Kua EH, Tan CH, Ng TP. Physical, social and productive leisure activities, cognitive decline and interaction with APOE-epsilon 4 genotype in Chinese older adults. Int Psychogeriatr. 2008 Apr;20(2): 237-51.

31 Gurău F, Baldoni S, Prattichizzo F, Espinosa E, Amenta F, Procopio AD, et al. Anti-senescence compounds: A potential nutraceutical approach to healthy aging. Ageing Res Rev. 2018 Sep;46:14-31.

32 Xiong LG, Chen YJ, Tong JW, Gong YS, Huang JA, Liu ZH. Epigallocatechin-3-gallate promotes healthy lifespan through mitohormesis during early-to-mid adulthood in Caenorhabditis elegans. Redox Biol. 2018 Apr;14: 305-15. 